\title{
DETECTION OF ORDERED AND CHAOTIC MOTION USING THE
}

\section{DYNAMICAL SPECTRA}

\author{
N. VOGLIS ${ }^{2}$, G. CONTOPOULOS ${ }^{1,2}$ and C. EFTHYMIOPOULOS ${ }^{1,2}$ \\ ${ }^{1}$ Research Center for Astronomy, Academy of Athens \\ ${ }^{2}$ Department of Astronomy, University of Athens
}

\begin{abstract}
Two simple and efficient numerical methods to explore the phase space structure are presented, based on the properties of the "dynamical spectra". 1) We calculate a "spectral distance" $D$ of the dynamical spectra for two different initial deviation vectors. $D \rightarrow 0$ in the case of chaotic orbits, while $D \rightarrow$ const $\neq 0$ in the case of ordered orbits. This method is by orders of magnitude faster than the method of the Lyapunov Characteristic Number (LCN). 2) We define a sensitive indicator called ROTOR (ROtational TOri Recongnizer) for 2D maps. The ROTOR remains zero in time on a rotational torus, while it tends to infinity at a rate $\propto N=$ number of iterations, in any case other than a rotational torus. We use this method to locate the last KAM torus of an island of stability, as well as the most important cantori causing stickiness near it.
\end{abstract}

\section{Introduction}

Let $\mathbf{R}=\left(x_{1}, x_{2}, \ldots, x_{n}\right)$ be the position vector and $\boldsymbol{\xi}=\left(d x_{1}, d x_{2}, \ldots, d x_{n}\right)$ be the deviation vector in the $\mathrm{n}$-dimensional phase space of a dynamical system. The evolution of these vectors can be given by the maps

$$
\mathbf{R}^{\prime}=\mathbf{F}(\mathbf{R} ; K), \quad \boldsymbol{\xi}^{\prime}=\frac{\partial \mathbf{F}}{\partial x} \boldsymbol{\xi}
$$

in time steps of $\Delta t$. A number of useful quantities can be defined in terms of these two vectors. Namely, we can define the 'stretching number'

$$
a=\frac{1}{\Delta t} \lim \ln \frac{\left|\boldsymbol{\xi}^{\prime}\right|}{|\boldsymbol{\xi}|}, \quad|\boldsymbol{\xi}| \rightarrow 0
$$

(Nicolis 1983,Fujisaka 1983, Froeschlé et al 1993, Voglis and Contopoulos 1994) which gives the current rate of deviation of nearby orbits. If $\boldsymbol{\xi}_{i j}=\left(d x_{i}, d x_{j}\right)$ is the projection of $\boldsymbol{\xi}$ on the plane $\left(x_{i}, x_{j}\right)$ we can define the 'helicity angle' $\Phi_{i j}$ that gives the orientation of the vector $\xi_{i j}$ with respect to the axis $x_{i}$, denoted by $\Phi_{i j}=\operatorname{ang}\left(x_{i}, \boldsymbol{\xi}_{i j}\right)$. The 'twist angle' $\phi_{i j}=\frac{1}{\Delta t} a n g\left(\boldsymbol{\xi}_{i j}, \boldsymbol{\xi}_{i j}^{\prime}\right)$ gives the current angular frequency of rotation of the vector $\boldsymbol{\xi}_{i j}$. Similar quantities can be defined also for the position vector, e.g. the rotation angle $\theta_{i j}=\frac{1}{\Delta t} \operatorname{ang}\left(\mathbf{R}_{i j}, \mathbf{R}_{i j}^{\prime}\right)$ gives the current angular frequency of rotation of the vector $\mathbf{R}_{i j}$.

If $Q$ is one of these quantities, i.e. $Q=a, \Phi_{i j}, \phi_{i j}, \theta_{i j} \ldots$ at the $k^{t h}$ iteration, we define as the 'dynamical spectrum of $Q$ ' the probability density of the values of $Q$ given by

$$
S(Q)=\frac{d N(Q, Q+d Q)}{N d Q}
$$

where $N$ is the total number of iterations and $d N(Q, Q+d Q)$ is the number of values of $Q$ in the interval $(Q, Q+d Q)$. 


\section{Detection of Ordered and Chaotic Orbits in 4D Maps}

The dynamical spectra can be used very efficiently to distinguish between ordered and chaotic motion. The following properties of invariance of the spectra $S(Q)$ are known: (Voglis and Contopoulos 1994, Contopoulos and Voglis 1996, 1997, Voglis and Efthymiopoulos 1998, Voglis et al. 1998):

1) The spectra $S(Q)$ are invariant with respect to the initial conditions $R_{0}$ along an orbit (invariance in time).

2) The spectra $S(Q)$ are invariant with respect to $\mathbf{R}_{0}$ and $\boldsymbol{\xi}_{0}$ provided that $\mathbf{R}_{0}$ belongs to a connected chaotic domain (invariant in space).

3) a) In 4D maps: If the motion is ordered, two different initial deviation vectors $\boldsymbol{\xi}_{01}, \boldsymbol{\xi}_{02}$ give different dynamical spectra, i.e.

$$
S\left(Q, \boldsymbol{\xi}_{01}\right) \neq S\left(Q, \boldsymbol{\xi}_{02}\right),
$$

while if the motion is chaotic they give the same spectrum

$$
S\left(Q, \boldsymbol{\xi}_{01}\right)=S\left(Q, \boldsymbol{\xi}_{02}\right) \text {. }
$$

3) b) In $2 D$ maps, the property $3 a$ is reduced to a similar property for the spectrum of the helicity angles only. Namely, if the motion is ordered, an opposite initial deviation vector gives a helicity spectrum shifted by $\pi$

$$
S\left(\Phi, \boldsymbol{\xi}_{0}\right)=S\left(\Phi+\pi,-\boldsymbol{\xi}_{0}\right),
$$

while if the motion is chaotic the two helicity spectra are equal and $\pi$-symmetric.

$$
S\left(\boldsymbol{\Phi}, \boldsymbol{\xi}_{0}\right)=S\left(\Phi,-\boldsymbol{\xi}_{0}\right)=S\left(\Phi+\pi, \boldsymbol{\xi}_{0}\right),
$$

The properties 3a,b are explained as follows. In 4D maps the ordered motion occurs on a $2 \mathrm{D}$ torus. Any initial deviation vector $\xi_{0}$ becomes tangent to this torus after a short transient period. In general, two different initial vectors $\boldsymbol{\xi}_{01}$ and $\boldsymbol{\xi}_{02}$ become tangent to different directions on the torus. Thus they produce different sequences of vectors $\xi_{1}$ and $\xi_{2}$ and hence different spectra.

On the other hand, two initially different deviation vectors of a chaotic orbit tend to coincide, after a few transient periods, on the direction defined by a nearby most unstable manifold. Thus, they produce the same dynamical spectra.

The convergence of the two deviation vectors is much faster than the convergence of the spectra to their final form (which happens in the same time scale as the stabilisation of the LCN to its constant value). Thus, by measuring the difference of the spectra we can recognise a chaotic orbit much faster than by calculating the LCN (or the final spectra).

We demonstrate the eficiency of the above method using the model of two coupled standard maps, i.e.

$$
\begin{array}{ll}
x_{1}^{\prime}=x_{1}+x_{2}^{\prime}, & x_{2}^{\prime}=x_{2}+\frac{K}{2 \pi} \sin 2 \pi x_{1}-\frac{\beta}{\pi} \sin 2 \pi\left(x_{3}-x_{1}\right) \\
x_{3}^{\prime}=x_{3}+x_{4}^{\prime}, & x_{4}^{\prime}=x_{4}+\frac{K}{2 \pi} \sin 2 \pi x_{3}-\frac{\beta}{\pi} \sin 2 \pi\left(x_{1}-x_{3}\right)
\end{array}
$$



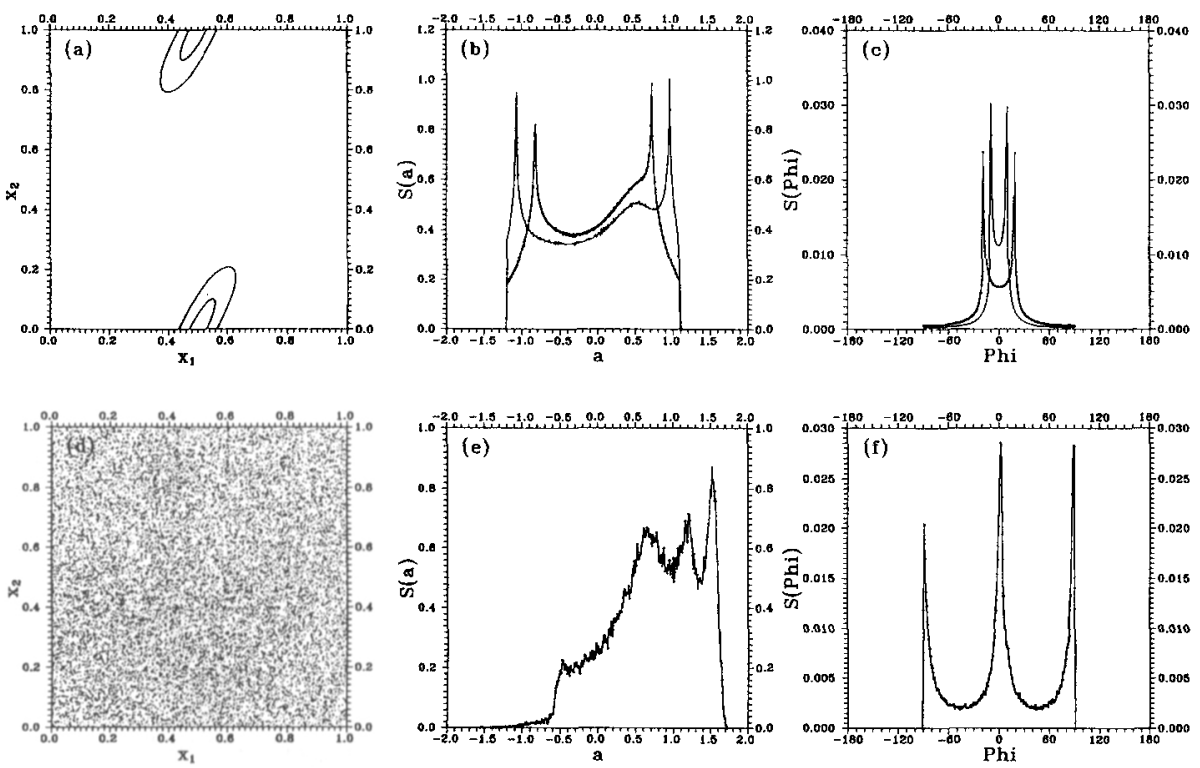

Fig. 1. Projections of the orbits (a) A1 and (d) B on the plane $\left(x_{1}, x_{2}\right)$. The respective spectra of $(b, e)$ stretching numbers and $(c, f)$ helicity angles.

For $K=3$ each uncoupled map $(\beta=0)$ has a phase portrait with one main island embedded in a chaotic sea. We select the following initial conditions: $\left(x_{1}, x_{2}, x_{3}, x_{4}\right)=(0.55,0.1,0.62,0.2)$ for three values of $\beta$, namely $\beta=10^{-5}$ (orbit A1), $\beta=10^{-1}$ (orbit A2), $\beta=0.3051$ (orbit A3), and $\left(x_{1}, x_{2}, x_{3}, x_{4}\right)=$ $(0.1,0.5,0.2,0.6)$, for $\beta=10^{-1}$ (orbit B). We iterate these orbits with two different initial deviation vectors, namely $\boldsymbol{\xi}_{1}=(1,1,1,1)$, and $\boldsymbol{\xi}_{2}=(2,2,1,1)$.

The projection of the orbit A1 on the plane $\left(x_{1}, x_{2}\right)$ gives two invariant curves (Fig.1a). This orbit, corresponding to two weakly coupled ordered 2D orbits, is ordered. On the other hand, orbit B is chaotic and its projection covers the plane $\left(x_{1}, x_{2}\right)$ uniformly (Fig. 1d).

The spectra of the stretching numbers $S(a)$ for the orbit A1 and the deviation vectors $\boldsymbol{\xi}_{1}$ and $\boldsymbol{\xi}_{2}$, after $10^{5}$ iterations, are shown in Fig. 1b. Both spectra are invariant in time, i.e. the next $10^{5}$ iterations produce the same spectra. However, the two spectra are clearly different from each other. The corresponding spectra of the helicity angles $S(\Phi)$ (Fig. 1c) are also different. In contrast, the spectra $S(a)$ and $S(\Phi)$ of the orbit B, and for the same two initial vectors $\xi_{1}, \xi_{2}$, coincide (Figs. 1e,f).

In order to quantify this behavior we define the distance $D$ between the two spectra of $Q$ ('spectral distance'), namely:

$$
D^{2}=\sum_{\text {all } Q}\left[S_{1}(Q)-S_{2}(Q)\right]^{2}
$$

In Fig. 2a the evolution of the finite time Lyapunov characteristic number 

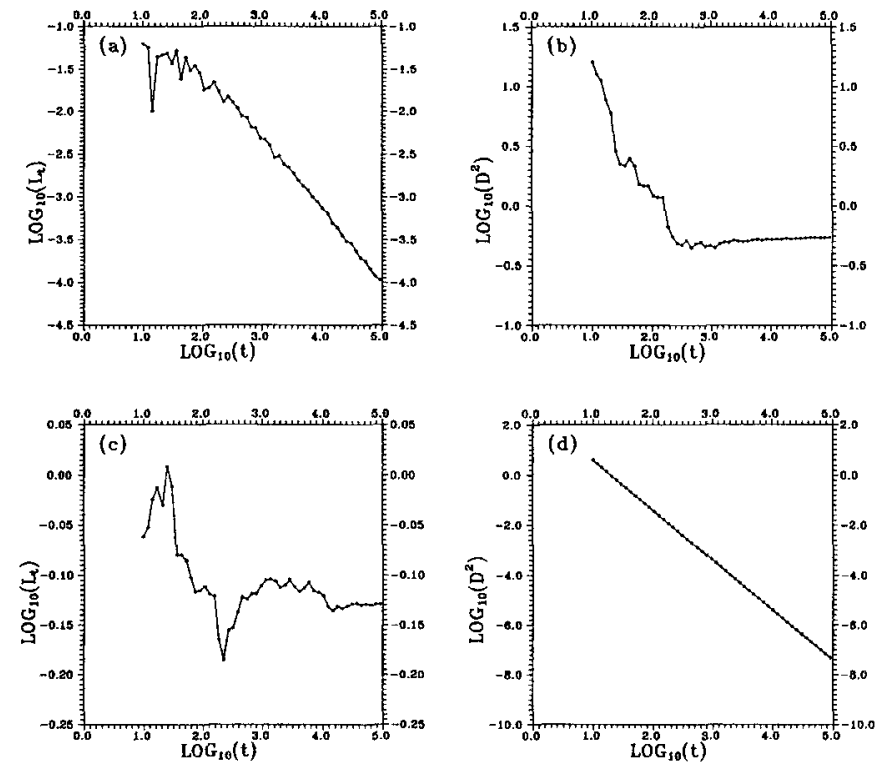

Fig. 2. The evolution of $(a, c)$ the finite time Lyapunov characteristic number and $(b, d)$ the spectral distance, for the orbits A1 and B.

$L_{t}$ is given for the orbit A1. As expected for regular orbits, $L_{t}$ decreases as $t^{-1}$, reaching the value $\approx 10^{-4}$ after $10^{5}$ iterations. The corresponding spectral distance of stretching numbers (fig. 2b), after a transient decrease for about $10^{2}$ periods, is stabilized near the value $D^{2} \approx 0.54$, showing that the two spectra $S(a)$ have constant difference.

On the other hand, the value of $L_{t}$ of the chaotic orbit B is stabilized at the value $\approx 0.75$ after $10^{5}$ iterations (fig. $2 \mathrm{c}$ ). The spectral distance $D$ (Fig. 2d) decreases at a constant logarithmic rate $\left(D^{2} \propto t^{-2}\right)$ and reaches the value of $10^{-2}$ after about 100 iterations, while it becomes smaller than $10^{-7}$ after $10^{5}$ iterations. Thus, the distinction between ordered and chaotic motion using $D^{2}$ is much faster than with the LCN criterion.

We apply now the method to the much more difficult case of the orbits A2 (regular) and A3 (chaotic with a very small LCN). The projections of these orbits on the plane $\left(x_{1}, x_{2}\right)$ are shown in Figs.3a,d. The projections of both orbits give the impression of motion on a torus. In particular, both projections remain in the same limited area even after $10^{9}$ iterations.

Despite their visual similarity, the orbit A2 is regular while the orbit A3 is chaotic. The character of the two orbits is revealed very efficiently using the spectral distance $D$. Namely, the two spectra $S(a)$ and $S(\Phi)$ for the orbit A2 (and $\xi_{1}, \xi_{2}$ as above) are different (Figs.3b,c), while those of the orbit A3 are identical (Figs. 3e,f).

The evolution of the finite time $\operatorname{LCN}\left(L_{t}\right)$ for both orbits is shown in Fig. 4. For 

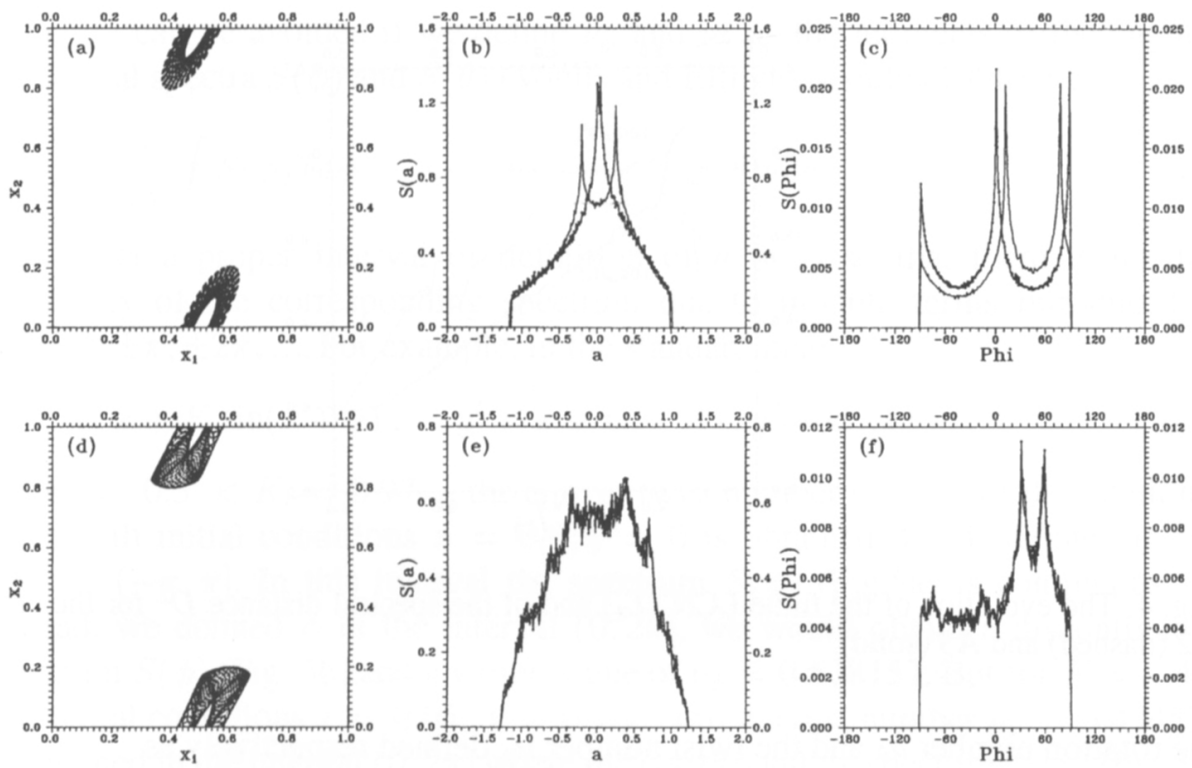

Fig. 3. Same as Fig.1 for the orbits A2 and A3.

the orbit A3 (solid line), $L_{t}$ converges to a very small positive value $4 \times 10^{-7}$ after $10^{9}$ iterations, while, for the orbit A2 (dashed line) $L_{t}$ continues falling as $t^{-1}$. In the same figure, the spectral distances $\log \left(D^{2}\right)$ for the two orbits (dashed for $A_{2}$, solid for $A_{3}$ ) after a transient phase of about $10^{3}$ iterations evolve in a different way. The spectral distance for the orbit $\mathrm{A} 2$ is stabilized to the value $D^{2} \approx 0.4$, while the spectral distance for the orbit A3 keeps decreasing, reaching a level of $10^{-3}$ after about $10^{5}$ iterations. The distinction of the two orbits A2 and A3 using $D^{2}$ is again much faster than with the finite time LCN.

Similar results are found in 3D Hamiltonian systems by considering either their spectra of stretching numbers along the flow or their 4D Poincare sections. In 2D maps, this method can be applied by calculating the spectra and spectral distances of the helicity angles.

\section{Detection of KAM Tori}

In this section we describe a method for 2D maps that detects KAM tori surrounding a central stable periodic orbit at a point $O\left(x_{0}, y_{0}\right)$ (rotational tori). The method is based on finding the average frequency of rotation of both the position vector $\mathbf{R}$ (with respect to $O$ ), and the deviation vector $\xi$ (Voglis 1996).

Two succesive vectors $\left(\mathbf{R}_{i}, \mathbf{R}_{i+1}\right)$ and $\left(\boldsymbol{\xi}_{i}, \boldsymbol{\xi}_{i+1}\right)$ define the rotation angle $\theta_{i}=$ $\operatorname{ang}\left(\mathbf{R}_{i}, \mathbf{R}_{i+1}\right)$ and the $t$ wist angle $\phi_{i}=\operatorname{ang}\left(\boldsymbol{\xi}_{i}, \boldsymbol{\xi}_{i+1}\right)$. The angles $\theta_{i}$ and $\phi_{i}$ give 


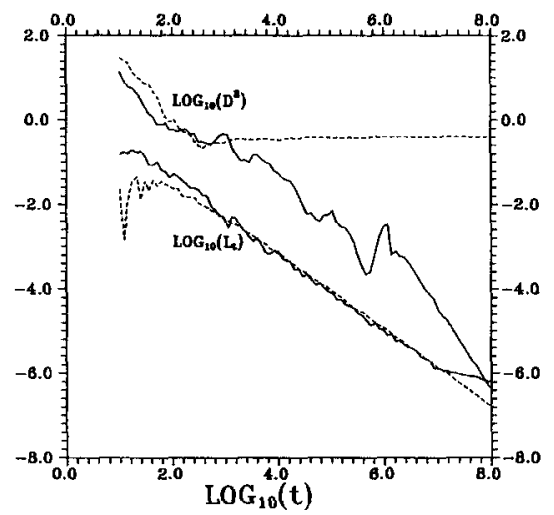

Fig. 4. The evolution of the finite LCN $\left(L_{t}\right)$ and of the spectral distance $D^{2}$ for the orbit A2 (dashed) and A3 (solid).

the rotation number $\nu_{\theta}$ and the twist number $\nu_{\phi}$ defined respectively as

$$
\nu_{\theta}=\lim \frac{\sum_{i=0}^{N} \theta_{i}}{2 \pi N}, \quad \nu_{\phi}=\lim \frac{\sum_{i=0}^{N} \phi_{i}}{2 \pi N}, \quad N \rightarrow \infty
$$

where $N$ in the number of iterations. The rotation and twist numbers $\nu_{\theta}$ and $\nu_{\phi}$ give the average frequency of rotation of the vectors $\mathbf{R}$ and $\xi$ around $O$. The difference $\nu_{\kappa}=\nu_{\phi}-\nu_{\theta}$ (epicyclic frequency) gives the average frequency of relative rotation of the vector $\boldsymbol{\xi}$ with respect to $\mathbf{R}$.

If the initial position vector $\mathbf{R}_{\mathbf{0}}$ belongs to a KAM torus around $O$ the epicyclic frequency is by definition zero, while it is non zero in all other cases (Voglis and Efthymiopoulos 1998). In fact, even in the first case, the limit $\nu_{\kappa}=0$ is reached only for $N \rightarrow \infty$, while the actual value of $\nu_{\kappa}$ for finite $N$ is given by

$$
\nu_{\kappa}=0+\delta / N, \quad 0<\delta<1 \text {. }
$$

We define the 'ROTOR' (acronym of 'ROtational TOri Recognizer') as the integer part of $N \nu_{\kappa}$, namely

$$
R O T O R \equiv r=\left[N \nu_{\kappa}\right]=\left[N\left(\nu_{\phi}-\nu_{\theta}\right)\right]=\left[\frac{\sum_{i=0}^{N}\left(\phi_{i}-\theta_{i}\right)}{2 \pi}\right] .
$$

The ROTOR is a function $r\left(\mathbf{R}_{0}, N\right)$ of the initial position vector and the current number of iterations. If $\mathbf{R}_{0}$ belongs to a KAM torus, then, as $N \rightarrow \infty$, the value of $r$ is constantly zero. Otherwise, $r$ tends to infinity proportionally to $N$, i.e.

$$
r\left(\mathbf{R}_{0}, N\right)= \begin{cases}0 & \text { if } \mathbf{R}_{0} \text { belongs to a KAM torus } \\ \propto N \rightarrow \infty & \text { otherwise }\end{cases}
$$

In practice, the correct evaluation of $\nu_{\phi}$ and $\nu_{\theta}$ as well as of the ROTOR is not a trivial task because of the multivalued character of the angles $\theta_{i}$ and $\phi_{i}$.This 
difficulty can be avoided if we define $\nu_{\phi}$ and $\nu_{\theta}$ as the moments of the angular dynamical spectra $S(\phi)$ and $S(\theta)$ (Voglis and Efthymiopoulos 1998), i.e.

$$
\nu_{\phi}=\frac{1}{2 \pi} \oint S(\phi) \phi d \phi, \quad \nu_{\theta}=\frac{1}{2 \pi} \oint S(\theta) \theta d \theta
$$

and select a proper interval of definition of $\theta$ or $\phi$ so that there is no discontinuity of the corresponding spectrum due to modulo terms introduced at $k \pi=0, \pm \pi, \pm 2 \pi, \ldots$. For example, in the standard map

$$
x^{\prime}=x+K \sin (x+y), \quad y^{\prime}=x+y, \quad(x, y \bmod 2 \pi)
$$

for $K=0.5<K_{c}=0.97 \ldots$, the correct twist number $\nu_{\phi}=0$ for the rotational torus with initial conditions $x=0.8, y=0$ is obtained if $\phi$ is defined in the interval $(-\pi, \pi]$. In this interval the spectrum $S(\phi)$ (Fig.5a) is continuous. If, instead, we defined $\phi$ in the interval $(0,2 \pi]$, we would obtain a discontinuous spectrum $S(\phi)$ (Fig. 5b) and a wrong value of $\nu_{\phi}=0.538157$. But for $K=-4.2$ and initial conditions $x=0.05, y=0$, the correct twist number $\nu_{\phi}=0.434576$ is obtained in the interval $(0,2 \pi]$ where the corresponding spectrum $S(\phi)$ (Fig.5d) is continuous. If we adopt the interval $(-\pi, \pi]$ (Fig.5c) we obtain a wrong value $\nu_{\phi}=0.042660$.

We apply now the ROTOR method in the standard map (Eqs. 16) for $K=-2.1$. In this case the phase portrait has one main island around the stable fixed point $(0,0)$. This island is surrounded by a chaotic sea. We calculate the ROTOR for orbits near the outer limit of the island scanning outwards along the diagonal $(x=y)$ with a step $d x=10^{-6}$ and $N_{\max }=10^{5}$ iterations for every orbit.

The dependence of the ROTOR on $x$ is shown in Fig. 6a. The right end of this figure (e.g. $x>0.7440$ ) is inside the chaotic sea. In this region the ROTOR is systematically far from zero. All the inverse U-shapes in this figure indicate first order islands, while the smaller U-shapes inside the first order islands correspond to second order islands (Voglis and Efthymiopoulos 1998).

Moving to the left in this figure (towards the center of the main island) we find the first place where the ROTOR has zero value at $x=0.743339$. The ROTOR maintains this zero value even after $10^{9}$ iterations. Thus, the ROTOR gives a good estimate of the position of the last KAM curve surrounding the main island.

In the region $0.7436<x<0.7438$ the ROTOR becomes small (near 1), but not zero. The closer approaches of the ROTOR to zero indicate orbits moving near cantori that are more efficient in producing stickiness. In Fig.6b the phase portrait near the last KAM curve is shown. There are only two orbits drawn in this figure: 1)the orbit with the above initial condition $x=y=0.743339$ (last KAM curve) and 2) a nearby chaotic orbit $(x=y=0.7436)$, which is sticky for about $9 \times 10^{8}$ periods before escaping to the chaotic sea. The darker regions in Fig. $6 \mathrm{~b}$ correspond to the position of cantori that limit the chaotic diffusion. In these darker regions the ROTOR has a value close to 1 . The positions of cantori can be located also in terms of their nearby periodic orbits (Efthymiopoulos et al. 1997). We find that 

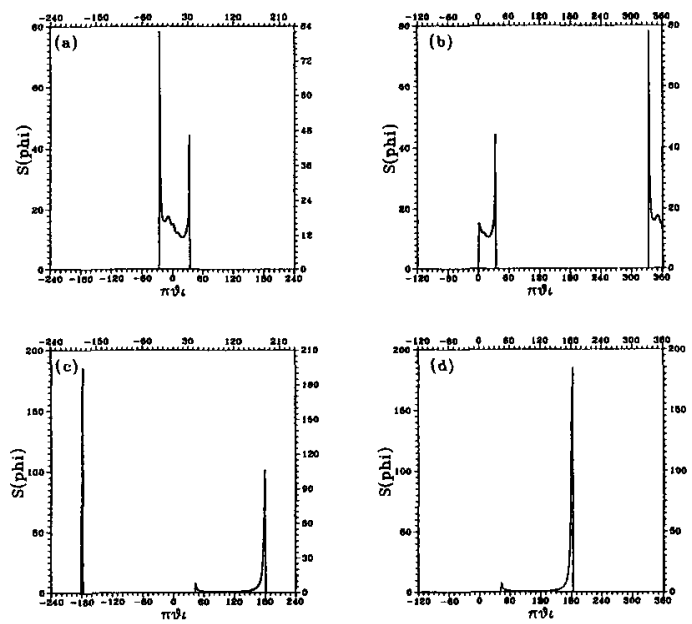

Fig. 5. The spectrum of twist angles $S(\phi)$ of the orbit with initial conditions $x=0.8, y=0$ in the standard map (Eq.16) for $K=0.5$ in the interval a) $(-\pi, \pi]$ and b) $[0,2 \pi)$.c,d) similar as a,b for the orbit $x=0.05, y=0$ and for $K=-4.2$.
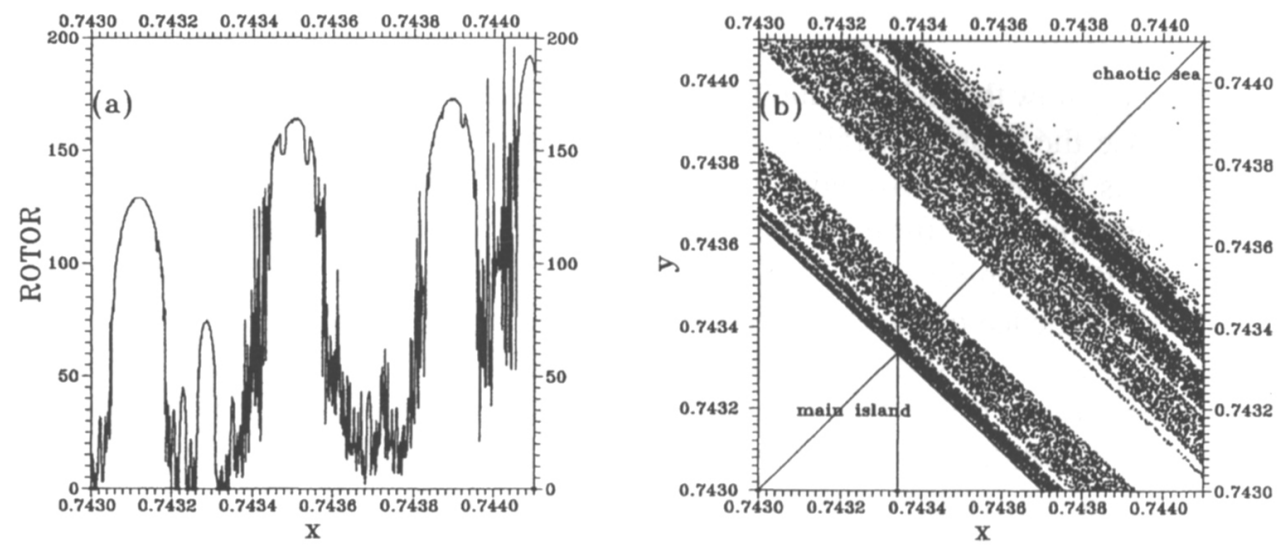

Fig. 6. a) The ROTOR as a function of $x$ in the standard map (Eq.16) along the scanning line $\mathrm{x}=\mathrm{y}$ from $x=0.743$ to $x=0.7441$. b) The phase portrait in the same region. The vertical line gives the last position where the ROTOR is zero. The intersection of this line with the diagonal $y=x$ marks the position of the last KAM torus.

the values of the ROTOR near 1 indicate the positions of the cantori with good accuracy.

\section{Discussion and Conclusions}

In this paper we present two simple numerical methods, based on the properties of dynamical spectra, to investigate the structure of phase space in dynamical systems. The first method (section 2) distinguishes between ordered and chaotic orbits by measuring the spectral distance $D$ for two deviation vectors from the same orbit. 
The spectral distance either becomes constant in time after a short transient period (ordered orbit), or decreases in time reaching a logarithmic slope $d \ln D / d \ln t=-1$ (chaotic orbit).

The second method (section 3 ) is based on a quantity called ROTOR, which is the integer part of the product of the epicyclic frequency $\nu_{\kappa}$ multiplied by the time $t$, i.e. $R O T O R=\left[t \nu_{\kappa}\right]$, where $t=N$, in a 2D map. If the ROTOR remains zero in time, the motion takes place on a KAM rotational torus. In the case of a secondary island the curve giving the ROTOR as a function of the initial conditions is U-shaped, or inverse U-shaped. In the case of chaos the curve is very irregular. This method is very sensitive because $t$ can be made arbitrarily large, thus the contrast between the values of ROTOR in rotational tori and in islands, or chaotic regions, can be made arbitrarily large.

In recent years various methods have been proposed for distinguishing between ordered and chaotic orbits. Such methods are based on the frequency analysis of the orbits, or on the deviations between nearby orbits.

Examples of methods of the first kind are:

1) the frequency analysis method of Laskar (1990) and Laskar et al. (1992)

2) the CLEANEST method of Foster $(1995,1996)$ and Gallardo and FerrazMello (1997)

3) the "Hénon method" of continued fraction approximations (Lega and Froeschlé 1996), and

4) the wavelet transform method (Arneodo et al. 1988, Bendjoya and Slezak 1993, Michtchenko and Ferraz-Mello 1995, Michtchenko and Nesvorny 1996).

Examples of methods of the second kind are:

1) the fast Lyapunov indicators method of Froeschlé et al. (1996), and

2) the methods of dynamical spectra (Voglis and Contopoulos 1994, Contopoulos and Voglis 1996, 1997, Voglis and Efthymiopoulos 1998, Voglis et al. 1998).

Every method has its own advantages. In the present paper we stress only the advantages of our methods. In particular, the methods presented here combine:

a) Speed. The method of spectral distance described in section 2 can distinguish between ordered and chaotic motion without the need of evaluating the fundamental frequencies of the orbits. It is based on a property of the variational equations that lose the memory of initial conditions exponentially fast in the case of chaotic motions. This property makes the method faster than the frequency analysis methods in which the convergence of the frequencies follows a power law.

b) Simplicity. As all the methods of dynamical spectra, the two methods presented here, namely the method of spectral distance and the method of ROTOR, require only the integration of an orbit and its variational equations.

c) Sensitivity and information content. Both our methods can be applied equally well in regions of strong or weak chaos. The spectral distance method gives a very sensitive distinction of whether the motion is on a torus or not, for systems of any number of degrees of freedom. This avoids the need to visualize the orbits on either the phase space or the frequency space. On the other hand, in the case of 2D systems, 
the method of angular dynamical spectra gives also the fundamental frequencies (rotation and epicyclic frequency). In particular, the evaluation of the epicyclic frequency allows a sharp distinction between islands and KAM tori. This makes the method particularly suitable in exploring the resonant structure (i.e. location of KAM tori,islands, and cantori) near the border of transition to stochasticity.

\section{Acknowledgements}

This research was supported in part by the Research Committee of the Academy of Athens (grant 200/409). C.E. was supported by the Greek Foundation of State Scholarships (IKY).

\section{References}

Arneodo, A., Grasseau, G., Holschneider, M.: 1988, Phys. Rev. Lett., 61, 2281.

Bendjoya, P. and Slezak, E.: 1993, Cel. Mech. Dyn. Astr, 56, 231.

Contopoulos, G., and Voglis, N.: 1996, Cel. Mech. Dyn. Astr., 64, 1.

Contopoulos, G., and Voglis, N.: 1997, Astron. Astrophys., 317, 73.

Efthymiopoulos, C., Contopoulos, G., Voglis, N., and Dvorak, R.: 1997, J. Phys., A 30, 8167.

Foster, G.: 1995, Astron. J., 109, 1889.

Foster, G.: 1996, Astron. J., 111, 541.

Froeschlé, C., Froeschlé, Ch., and Lohinger, E.: 1993, Cel. Mech. Dyn. Astron., 56, 307.

Froeschlé, C., Lega, E. and Gonczi, R.: 1997, Cel. Mech. Dyn. Astron., 67, 41.

Fujisaka, H.: 1983, Prog. Theor. Phys., 70, 1264.

Gallardo, T. and Ferraz-Mello, S.: 1997, Astron. J., 113, 863.

Laskar, J.:1990, Icarus 88, 266.

Laskar, J., Froeschlé, C. and Celletti, A.: 1993, Physica, D56, 253.

Lega, E. and Froeschlé, C.: 1996, Physica, D95, 97.

Michtchenko, T.A. and Ferraz-Mello, S.:1995 Astron. Astrophys., 303, 945.

Michtchenko, T.A. and Nesvorny, D.: 1996, Astron. Astrophys., 313, 674.

Nicolis, J.S., Meyer-Kress, G., and Haubs, G.: 1983, Z.Naturfosch., 38a, 1157.

Voglis, N.:1996, Human Capital and Mobility Workshop, Santorini, Greece (oral presentation).

Voglis, N., and Contopoulos, G.: 1994, J. Phys., A 27, 4899.

Voglis, N., and Efthymiopoulos, C.: 1998, J. Phys., A 31, 2913.

Voglis, N., Contopoulos, G., and Efthymiopoulos, C.: 1998, Phys. Rev. , E 57, 372. 